\title{
Going Beyond the "Whole Wetland": Small-Scale Within-Wetland Heterogeneity, Translates To Big Difference in Methane Flux Dynamics and Regulation
}

\author{
Gil Bohrer \\ The Ohio State University, USA \\ bohrer.17@osu.edu
}

\begin{abstract}
In the global context, wetlands are small. However, wetlands are responsible for a very large fraction of the global methane flux, the second most effective greenhouse gas. Current modeling efforts and global upscaling of observations of wetland methane fluxes assume the whole-wetland as the atomic scale of their resolution. Observations are averaged to represent the whole wetland where they were taken, and applied to other wetlands of a similar type and climate. Models represent a single wetland-patch within a resolved land-surface pixel and predict the averaged fluxes from that wetland patch based on the meteorological conditions, ignoring within-wetland heterogeneity of hydrology and ecology. However, wetlands are typically highly heterogeneous, and observations showed large differences in flux rates from different vegetation patches within the same wetlands. We used multi-scale observations approach, combining eddy covariance, and surface and leaf chamber observations of methane fluxes, and vertically detailed pore water concentrations of dissolved methane and genetic characterization of the microbiomes. The wetland where we work has extremely high methane fluxes, but it is not untypical for wetlands in agricultural watersheds in the Midwest. We demonstrate that any sampling of methane fluxes in a wetland must account for the areas of the eco-hydrological patch types that each of the samples within the wetland represent. We observed the methane paradox, where high methane production rates are present in the shallow and bulkaerobic soil layer. We provide process-level observations of the parameters and dynamics that govern methane production and emission. We show different patterns of regulation of methane transport through plants, and provide direct observations of the bulk conductivity of different vegetation types at different times through the season. The parameters and processes we observe could be incorporated in land-surface models that simulate wetlands at the resolution of eco-hydrological subpatches within wetlands, to improve the global predictability of methane fluxes.
\end{abstract}

